\title{
Research Article \\ Projection Methods and a New System of Extended General Regularized Nonconvex Set-Valued Variational Inequalities
}

\author{
Javad Balooee, ${ }^{1}$ Yeol Je Cho, ${ }^{2}$ and Mee Kwang Kang ${ }^{3}$ \\ ${ }^{1}$ Department of Mathematics, Ayatollah Amoli Branch, Islamic Azad University, Amol, Iran \\ ${ }^{2}$ Department of Mathematics Education and RINS, Gyeongsang National University, \\ Chinju 660-701, Republic of Korea \\ ${ }^{3}$ Department of Mathematics, Dongeui University, Pusan 614-714, Republic of Korea \\ Correspondence should be addressed to Yeol Je Cho, yjcho@gnu.ac.kr \\ and Mee Kwang Kang, mee@dongeui.ac.kr
}

Received 2 October 2011; Accepted 11 November 2011

Academic Editor: Rudong Chen

Copyright (C) 2012 Javad Balooee et al. This is an open access article distributed under the Creative Commons Attribution License, which permits unrestricted use, distribution, and reproduction in any medium, provided the original work is properly cited.

\begin{abstract}
A new system of extended general nonlinear regularized nonconvex set-valued variational inequalities is introduced, and the equivalence between the extended general nonlinear regularized nonconvex set-valued variational inequalities and the fixed point problems is verified. Then, by this equivalent formulation, a new perturbed projection iterative algorithm with mixed errors for finding a solution of the aforementioned system is suggested and analyzed. Also the convergence of the suggested iterative algorithm under some suitable conditions is proved.
\end{abstract}

\section{Introduction}

Variational inequality theory, introduced by Stampacchia [1], has become a rich source of inspiration and motivation for the study of a large number of problems arising in economics, finance, transportation, network and structural analysis, elasticity, and optimization. Many research papers have been written lately, both on the theory and applications of this field. Important connections with main areas of pure and applied sciences have been made. (See, for example, [2-4] and the references cited therein.) The development of variational inequality theory can be viewed as the simultaneous pursuit of two different lines of research. On the one hand, it reveals the fundamental facts on the qualitative aspects of the solution to important classes of problems. On the other hand, it also enables us to develop highly efficient and powerful new numerical methods to solve, for example, obstacle, unilateral, free, moving 
and the complex equilibrium problems. One of the most interesting and important problems in variational inequality theory is the development of an efficient numerical method. There is a substantial number of numerical methods including projection method and its variant forms, Wiener-Holf (normal) equations, auxiliary principle, and descent framework for solving variational inequalities and complementarity problems. For applications on physical formulations, numerical methods and other aspects of variational inequalities, see [1-37] and the references therein.

Projection method and its variant forms represent important tool for finding the approximate solution of various types of variational and quasi-variational inequalities, the origin of which can be traced back to Lions and Stampacchia [23]. The projection type methods were developed in 1970s and 1980s. The main idea in this technique is to establish the equivalence between the variational inequalities and the fixed point problem using the concept of projection. This alternate formulation enables us to suggest some iterative methods for computing the approximate solution; for example, see $[5-7,16-18,29,30,35-$ 37].

It should be pointed that almost all the results regarding the existence and iterative schemes for solving variational inequalities and related optimizations problems are being considered in the convexity setting. Consequently, all the techniques are based on the properties of the projection operator over convex sets, which may not hold in general, when the sets are nonconvex. It is known that the uniformly prox-regular sets are nonconvex and include the convex sets as special cases. For more details, see, for example, $[12,20$, 21, 28]. In recent years, Bounkhel et al. [12], Moudafi [24], Noor [25, 26], and Pang et al. [27] have considered variational inequalities in the context of uniformly prox-regular sets.

In this paper, we introduce and consider a new system of extended general nonlinear regularized nonconvex set-valued variational inequalities. We establish the equivalence between the extended general nonlinear regularized nonconvex set-valued variational inequalities and the fixed point problems, and then, by this equivalent formulation, we suggest and analyze a new perturbed projection iterative algorithm with mixed errors for finding a solution of the aforementioned system. We also prove the convergence of the suggested iterative algorithm under some suitable conditions.

\section{Preliminaries}

Throughout this paper, we will let $\mathscr{t}$ be a real Hilbert space which is equipped with an inner product $\langle\cdot, \cdot\rangle$ and corresponding norm $\|\cdot\|$. Let $K$ be a nonempty convex subset of $\mathscr{d}$, and, $C B(\mathscr{L})$ denote the family of all closed and bounded subsets of $\mathscr{L}$. We denote by $d_{K}(\cdot)$ or $d(\cdot, K)$ the usual distance function to the subset $K$; that is, $d_{K}(u)=\inf _{v \in K}\|u-v\|$. Let us recall the following well-known definitions and some auxiliary results of nonlinear convex analysis and nonsmooth analysis [19-21,28].

Definition 2.1. Let $u \in \mathcal{H}$ be a point not lying in $K$. A point $v \in K$ is called a closest point or a projection of $u$ onto $K$ if $d_{K}(u)=\|u-v\|$. The set of all such closest points is denoted by $P_{K}(u)$; that is,

$$
P_{K}(u):=\left\{v \in K: d_{K}(u)=\|u-v\|\right\} .
$$


Definition 2.2. The proximal normal cone of $K$ at a point $u \in \mathscr{H}$ with $u \notin K$ is given by

$$
N_{K}^{P}(u):=\left\{\xi \in \mathscr{d}: u \in P_{K}(u+\alpha \xi) \text {, for some } \alpha>0\right\} .
$$

Clarke et al. [20], in Proposition 1.1.5, give a characterization of $N_{K}^{P}(u)$ as the following.

Lemma 2.3. Let $K$ be a nonempty closed subset in $\mathscr{d e}$. Then $\xi \in N_{K}^{P}(u)$ if and only if there exists a constant $\alpha=\alpha(\xi, u)>0$ such that $\langle\xi, v-u\rangle \leq \alpha\|v-u\|^{2}$ for all $v \in K$.

The above inequality is called the proximal normal inequality. The special case in which $K$ is closed and convex is an important one. In Proposition 1.1.10 of [20], the authors give the following characterization of the proximal normal cone, the closed and convex subset $K \subset \mathfrak{l}$.

Lemma 2.4. Let $K$ be a nonempty, closed, and convex subset in $\mathcal{L l}$. Then $\xi \in N_{K}^{P}(u)$ if and only if $\langle\xi, v-u\rangle \leq 0$, for all $v \in K$.

Definition 2.5. Let $X$ be a real Banach space, and let $f: X \rightarrow \mathbb{R}$ be the Lipschitz with constant $\tau$ near a given point $x \in X$; that is, for some $\varepsilon>0$, one has $|f(y)-f(z)| \leq \tau\|y-z\|$ for all $y, z \in B(x ; \varepsilon)$ where $B(x ; \varepsilon)$ denotes the open ball of radius $r>0$ and centered at $x$. The generalized directional derivative of $f$ at $x$ in the direction $v$, denoted as $f^{\circ}(x ; v)$, is defined as follows:

$$
f^{\circ}(x ; v)=\limsup _{y \rightarrow x, t \downarrow 0} \frac{f(y+t v)-f(y)}{t}
$$

where $y$ is a vector in $X$ and $t$ is a positive scalar.

The generalized directional derivative defined earlier can be used to develop a notion of tangency that does not require $K$ to be smooth or convex.

Definition 2.6. The tangent cone $T_{K}(x)$ to $K$ at a point $x$ in $K$ is defined as follows:

$$
T_{K}(x):=\left\{v \in \mathscr{\ell}: d_{K}^{\circ}(x ; v)=0\right\} .
$$

Having defined a tangent cone, the likely candidate for the normal cone is the one obtained from $T_{K}(x)$ by polarity. Accordingly, we define the normal cone of $K$ at $x$ by polarity with $T_{K}(x)$ as follows:

$$
N_{K}(x):=\left\{\xi:\langle\xi, v\rangle \leq 0, \forall v \in T_{K}(x)\right\}
$$

Definition 2.7. The Clarke normal cone, denoted by $N_{K}^{C}(x)$, is given by $N_{K}^{C}(x)=\overline{\mathrm{co}}\left[N_{K}^{P}(x)\right]$, where $\overline{c o}[S]$ means the closure of the convex hull of $S$. It is clear that one always has $N_{K}^{P}(x) \subseteq$ $N_{K}^{C}(x)$. The converse is not true in general. Note that $N_{K}^{C}(x)$ is always closed and convex cone, whereas $N_{K}^{P}(x)$ is always convex but may not be closed (see $[19,20,28]$ ).

In 1995, Clarke et al. [21] introduced and studied a new class of nonconvex sets, called proximally smooth sets; subsequently, Poliquin et al. in [28] investigated the aforementioned 
sets, under the name of uniformly prox-regular sets. These have been successfully used in many nonconvex applications in areas such as optimizations, economic models, dynamical systems, differential inclusions, and so forth. For such applications see [9-11, 13]. This class seems particularly well suited to overcome the difficulties which arise due to the nonconvexity assumptions on $K$. We take the following characterization proved in [21] as a definition of this class. We point out that the original definition was given in terms of the differentiability of the distance function (see [21]).

Definition 2.8. For any $r \in(0,+\infty]$, a subset $K_{r}$ of $\mathscr{\ell}$ is called normalized uniformly prox-regular (or uniformly r-prox-regular [21]) if every nonzero proximal normal to $K_{r}$ can be realized by an $r$-ball.

This means that, for all $\bar{x} \in K_{r}$ and all $0 \neq \xi \in N_{K_{r}}^{P}(\bar{x})$ with $\|\xi\|=1$,

$$
\langle\xi, x-\bar{x}\rangle \leq \frac{1}{2 r}\|x-\bar{x}\|^{2}, \quad \forall x \in K_{r} .
$$

Obviously, the class of normalized uniformly prox-regular sets is sufficiently large to include the class of convex sets, $p$-convex sets, and $C^{1,1}$ submanifolds (possibly with boundary) of $\mathscr{d}$, the images under a $C^{1,1}$ diffeomorphism of convex sets and many other nonconvex sets, see $[14,21]$.

Lemma 2.9 (see [21]). A closed set $K \subseteq \mathscr{H}$ is convex if and only if it is proximally smooth of radius $r$ for every $r>0$.

If $r=+\infty$, then, in view of Definition 2.8 and Lemma 2.9, the uniform $r$-prox-regularity of $K_{r}$ is equivalent to the convexity of $K_{r}$, which makes this class of great importance. For the case of that $r=+\infty$, we set $K_{r}=K$.

The following proposition summarizes some important consequences of the uniform prox-regularity needed in the sequel. The proof of this results can be found in [21, 28].

Proposition 2.10. Let $r>0$, and let $K_{r}$ be a nonempty closed and uniformly $r$-prox-regular subset of $\mathscr{H}$. Set $U(r)=\left\{u \in \mathscr{L}: 0<d_{K_{r}}(u)<r\right\}$. Then the following statements hold.

(a) For all $x \in U(r)$, one has $P_{K_{r}}(x) \neq \emptyset$.

(b) For all $r^{\prime} \in(0, r), P_{K_{r}}$ is Lipschitz continuous with constant $r /\left(r-r^{\prime}\right)$ on $U\left(r^{\prime}\right)=\{u \in$ de: $\left.0<d_{K_{r}}(u)<r^{\prime}\right\}$.

(c) The proximal normal cone is closed as a set-valued mapping.

As a direct consequent of part (c) of Proposition 2.10, we have $N_{K_{r}}^{C}(x)=N_{K_{r}}^{P}(x)$. Therefore, we will define $N_{K_{r}}(x):=N_{K_{r}}^{C}(x)=N_{K_{r}}^{P}(x)$ for such a class of sets.

In order to make clear the concept of $r$-prox-regular sets, we state the following concrete example. The union of two disjoint intervals $[a, b]$ and $[c, d]$ is $r$-prox-regular with $r=(c-b) / 2$. The finite union of disjoint intervals is also $r$-prox-regular, and $r$ depends on the distances between the intervals. 
Definition 2.11. The single-valued operator $h: \mathscr{d} \rightarrow \mathscr{H}$ is called

(a) monotone if

$$
\langle h(x)-h(y), x-y\rangle \geq 0, \quad \forall x, y \in \mathscr{H},
$$

(b) $r$-strongly monotone if, there exists a constant $r>0$ such that

$$
\langle h(x)-h(y), x-y\rangle \geq r\|x-y\|^{2}, \quad \forall x, y \in \mathscr{H},
$$

(c) $\gamma$-Lipschitz continuous if there exists a constant $\gamma>0$ such that

$$
\|h(x)-h(y)\| \leq r\|x-y\|, \quad \forall x, y \in \mathscr{\ell} .
$$

Definition 2.12. Let $T: \mathscr{d} \multimap \mathscr{l}$ be a set-valued operator, and let $g: \mathscr{t} \rightarrow \mathscr{d}$ be a singlevalued operator. Then $T$ is said to be

(a) monotone if

$$
\langle u-v, x-y\rangle \geq 0, \quad \forall x, y \in \mathscr{l}, u \in T(x), v \in T(y)
$$

(b) $\kappa$-strongly monotone with respect to $g$ if there exists a constant $\kappa>0$ such that

$$
\langle u-v, g(x)-g(y)\rangle \geq \kappa\|x-y\|^{2}, \quad \forall x, y \in \mathscr{H}, u \in T(x), v \in T(y) .
$$

Definition 2.13. A two-variable set-valued operator $T: \mathscr{H} \times \mathscr{H} \multimap \mathscr{H}$ is called $\xi$ - $\widehat{H}$-Lipschitz continuous in the first variable, if there exists a constant $\xi>0$ such that, for all $x, x^{\prime} \in \mathscr{d}$,

$$
\widehat{H}\left(T(x, y), T\left(x^{\prime}, y^{\prime}\right)\right) \leq \xi\left\|x-x^{\prime}\right\|, \quad \forall y, y^{\prime} \in \mathscr{l},
$$

where $\widehat{H}$ is the Hausdorff pseudo-metric, that is, for any two nonempty subsets $A$ and $B$ of th,

$$
\widehat{H}(A, B)=\max \left\{\sup _{x \in A} d(x, B), \sup _{y \in B} d(y, A)\right\} .
$$

It should be pointed that if the domain of $\widehat{H}$ is restricted to the family closed bounded subsets of $\mathscr{H}$ (denoted by $C B(\mathscr{\ell})$ ), then $\widehat{H}$ is the Hausdorff metric. 


\section{System of Extended General Regularized Nonconvex Set-Valued Variational Inequalities}

In this section, we introduce a new system of extended general nonlinear regularized nonconvex set-valued variational inequalities and a new system of extended general nonlinear set-valued variational inequalities in Hilbert spaces and investigate their relations.

Let $T_{i}: \mathscr{H} \times \mathscr{H} \rightarrow C B(\mathscr{l})(i=1,2)$ be two nonlinear set-valued operators, and let $g_{i}, h_{i}: \mathscr{H} \rightarrow \mathscr{H}(i=1,2)$ be four nonlinear single-valued operators such that $K_{r} \subseteq g_{i}(\mathscr{\ell})$, for each $i=1,2$. For any constants $\rho>0$ and $\eta>0$, we consider the problem of finding $x^{*}, y^{*} \in \mathscr{H}$ and $u^{*} \in T_{1}\left(y^{*}, x^{*}\right), w^{*} \in T_{2}\left(x^{*}, y^{*}\right)$ such that $h_{1}\left(x^{*}\right), h_{2}\left(y^{*}\right) \in K_{r}$ and

$$
\begin{aligned}
& \left\langle\rho u^{*}+h_{1}\left(x^{*}\right)-g_{1}\left(y^{*}\right), g_{1}(x)-h_{1}\left(x^{*}\right)\right\rangle+\frac{1}{2 r}\left\|g_{1}(x)-h_{1}\left(x^{*}\right)\right\|^{2} \geq 0, \quad \forall x \in \mathscr{H}: g_{1}(x) \in K_{r}, \\
& \left\langle\eta w^{*}+h_{2}\left(y^{*}\right)-g_{2}\left(x^{*}\right), g_{2}(x)-h_{2}\left(y^{*}\right)\right\rangle+\frac{1}{2 r}\left\|g_{2}(x)-h_{2}\left(y^{*}\right)\right\|^{2} \geq 0, \quad \forall x \in \mathscr{H}: g_{2}(x) \in K_{r} .
\end{aligned}
$$

The problem (3.1) is called the system of extended general nonlinear regularized nonconvex set-valued variational inequalities involving six different nonlinear operators.

Lemma 3.1. If $K_{r}$ is a uniformly prox-regular set, then the problem (3.1) is equivalent to that of finding $x^{*}, y^{*} \in \mathcal{H}$ and $u^{*} \in T_{1}\left(y^{*}, x^{*}\right), w^{*} \in T_{2}\left(x^{*}, y^{*}\right)$ such that $h_{1}\left(x^{*}\right), h_{2}\left(y^{*}\right) \in K_{r}$ and

$$
\begin{gathered}
0 \in \rho u^{*}+h_{1}\left(x^{*}\right)-g_{1}\left(y^{*}\right)+N_{K_{r}}^{P}\left(h_{1}\left(x^{*}\right)\right) \\
0 \in \eta w^{*}+h_{2}\left(y^{*}\right)-g_{2}\left(x^{*}\right)+N_{K_{r}}^{P}\left(h_{2}\left(y^{*}\right)\right)
\end{gathered}
$$

where $N_{K_{r}}^{P}(s)$ denotes the P-normal cone of $K_{r}$ at $s$ in the sense of nonconvex analysis.

Proof. Let $\left(x^{*}, y^{*}, u^{*}, w^{*}\right)$ with $x^{*}, y^{*} \in \mathscr{L}, h_{1}\left(x^{*}\right), h_{2}\left(y^{*}\right) \in K_{r}$, and $u^{*} \in T_{1}\left(y^{*}, x^{*}\right), w^{*} \in$ $T_{2}\left(x^{*}, y^{*}\right)$ be a solution of the system (3.1). If $\rho u^{*}+h_{1}\left(x^{*}\right)-g_{1}\left(y^{*}\right)=0$, because the vector zero always belongs to any normal cone, then $0 \in \rho u^{*}+h_{1}\left(x^{*}\right)-g_{1}\left(y^{*}\right)+N_{K_{r}}^{P}\left(h_{1}\left(x^{*}\right)\right)$. If $\rho u^{*}+h_{1}\left(x^{*}\right)-g_{1}\left(y^{*}\right) \neq 0$, then, for all $x \in \mathscr{L}$ with $g_{1}(x) \in K_{r}$, one has

$$
\left\langle-\left(\rho u^{*}+h_{1}\left(x^{*}\right)-g_{1}\left(y^{*}\right)\right), g_{1}(x)-h_{1}\left(x^{*}\right)\right\rangle \leq \frac{1}{2 r}\left\|g_{1}(x)-h_{1}\left(x^{*}\right)\right\|^{2}
$$

Now, by Lemma 2.3, one gets $-\left(\rho u^{*}+h_{1}\left(x^{*}\right)-g_{1}\left(y^{*}\right)\right) \in N_{K_{r}}^{P}\left(h_{1}\left(x^{*}\right)\right)$, and so

$$
0 \in \rho u^{*}+h_{1}\left(x^{*}\right)-g_{1}\left(y^{*}\right)+N_{K_{r}}^{P}\left(h_{1}\left(x^{*}\right)\right)
$$

Similarly, one can establish

$$
0 \in \eta w^{*}+h_{2}\left(y^{*}\right)-g_{2}\left(x^{*}\right)+N_{K_{r}}^{P}\left(h_{2}\left(y^{*}\right)\right) .
$$


Conversely, if $\left(x^{*}, y^{*}, u^{*}, w^{*}\right)$ with $x^{*}, y^{*} \in \mathscr{L}, h_{1}\left(x^{*}\right), h_{2}\left(y^{*}\right) \in K_{r}$, and $u^{*} \in T_{1}\left(y^{*}, x^{*}\right), w^{*} \in$ $T_{2}\left(x^{*}, y^{*}\right)$ is a solution of the system (3.2), then, in view of Definition $2.8, x^{*}, y^{*} \in \mathscr{d}$ and $u^{*} \in T_{1}\left(y^{*}, x^{*}\right), w^{*} \in T_{2}\left(x^{*}, y^{*}\right)$ with $h_{1}\left(x^{*}\right), h_{2}\left(y^{*}\right) \in K_{r}$ are a solution of the system (3.1).

The problem (3.2) is called the extended general nonlinear nonconvex set-valued variational inclusion system associated with the system of extended general nonlinear regularized nonconvex set-valued variational inequalities (3.1).

Some special cases of the system (3.1) are as follows.

Case 1. If $r=\infty$; that is, $K_{r}=K$, the convex set in $\mathscr{\ell}$, then the system (3.1) collapses to the following system.

Find $x^{*}, y^{*} \in \mathscr{H}$ and $u^{*} \in T_{1}\left(y^{*}, x^{*}\right), w^{*} \in T_{2}\left(x^{*}, y^{*}\right)$ such that $h_{1}\left(x^{*}\right), h_{2}\left(y^{*}\right) \in K$ and

$$
\begin{gathered}
\left\langle\rho u^{*}+h_{1}\left(x^{*}\right)-g_{1}\left(y^{*}\right), g_{1}(x)-h_{1}\left(x^{*}\right)\right\rangle \geq 0, \quad \forall x \in \mathscr{H}: g_{1}(x) \in K, \\
\left\langle\eta w^{*}+h_{2}\left(y^{*}\right)-g_{2}\left(x^{*}\right), g_{2}(x)-h_{2}\left(y^{*}\right)\right\rangle \geq 0, \quad \forall x \in \mathscr{H}: g_{2}(x) \in K,
\end{gathered}
$$

which is called the system of extended general nonlinear set-valued variational inequalities in the sense of convex analysis.

Case 2. If $T_{1}, T_{2}: \mathscr{H} \times \mathscr{H} \rightarrow \mathscr{H}$ are two nonlinear single-valued operators, $h_{i} \equiv I$, the identity operator, and $g_{i}=g(i=1,2)$, then the system (3.6) reduces to the system of finding $x^{*}, y^{*} \in K$ such that

$$
\begin{aligned}
& \left\langle\rho T_{1}\left(y^{*}, x^{*}\right)+x^{*}-g\left(y^{*}\right), g(x)-x^{*}\right\rangle \geq 0, \quad \forall x \in \mathscr{H}: g(x) \in K, \\
& \left\langle\eta T_{2}\left(x^{*}, y^{*}\right)+y^{*}-g\left(x^{*}\right), g(x)-y^{*}\right\rangle \geq 0, \quad \forall x \in \mathscr{H}: g(x) \in K,
\end{aligned}
$$

which has been considered and studied by Noor [26].

Case 3. If $r=\infty$; that is, $K_{r}=K, T_{1}, T_{2}: \mathscr{H} \rightarrow \mathscr{H}$ are two univariate nonlinear singlevalued operators, and $h_{i}=g_{i}=g(i=1,2)$, then the system (3.1) changes into that of finding $x^{*}, y^{*} \in K$ such that $g\left(x^{*}\right), g\left(y^{*}\right) \in K$ and

$$
\begin{aligned}
& \left\langle\rho T_{1}\left(y^{*}\right)+g\left(x^{*}\right)-g\left(y^{*}\right), g(x)-g\left(x^{*}\right)\right\rangle \geq 0, \quad \forall x \in \mathscr{H}: g(x) \in K, \\
& \left\langle\eta T_{2}\left(x^{*}\right)+g\left(y^{*}\right)-g\left(x^{*}\right), g(x)-g\left(y^{*}\right)\right\rangle \geq 0, \quad \forall x \in \mathscr{L}: g(x) \in K,
\end{aligned}
$$

which has been introduced and studied by Yang et al. [34].

Case 4. If $T_{1}=T_{2}=T$, then the problem (3.7) is equivalent to finding $x^{*}, y^{*} \in K$ such that

$$
\begin{aligned}
& \left\langle\rho T\left(y^{*}, x^{*}\right)+x^{*}-g\left(y^{*}\right), g(x)-x^{*}\right\rangle \geq 0, \quad \forall x \in \mathscr{H}: g(x) \in K, \\
& \left\langle\eta T\left(x^{*}, y^{*}\right)+y^{*}-g\left(x^{*}\right), g(x)-y^{*}\right\rangle \geq 0, \quad \forall x \in \mathscr{H}: g(x) \in K,
\end{aligned}
$$

which was considered and investigated by Noor [26]. 
Case 5. If $g \equiv I$, then the system (3.7) reduces to the system of finding $x^{*}, y^{*} \in K$ such that

$$
\begin{aligned}
& \left\langle\rho T_{1}\left(y^{*}, x^{*}\right)+x^{*}-y^{*}, x-x^{*}\right\rangle \geq 0, \quad \forall x \in K, \\
& \left\langle\eta T_{2}\left(x^{*}, y^{*}\right)+y^{*}-x^{*}, x-y^{*}\right\rangle \geq 0, \quad \forall x \in K,
\end{aligned}
$$

which has been considered and studied by Huang and Noor [22].

Case 6. If $g \equiv I$, then the system (3.9) changes into that of finding $x^{*}, y^{*} \in K$ such that

$$
\begin{array}{ll}
\left\langle\rho T\left(y^{*}, x^{*}\right)+x^{*}-y^{*}, x-x^{*}\right\rangle \geq 0, & \forall x \in K, \\
\left\langle\eta T\left(x^{*}, y^{*}\right)+y^{*}-x^{*}, x-y^{*}\right\rangle \geq 0, & \forall x \in K .
\end{array}
$$

The system (3.11) has been studied and investigated by Chang et al. [15] and Verma [33].

Case 7. If $T: \mathscr{l} \rightarrow \mathscr{H}$ is an univariate nonlinear operator, then the system (3.11) reduces to the following system: find $x^{*}, y^{*} \in K$ such that

$$
\begin{aligned}
& \left\langle\rho T\left(y^{*}\right)+x^{*}-y^{*}, x-x^{*}\right\rangle \geq 0, \quad \forall x \in K, \\
& \left\langle\eta T\left(x^{*}\right)+y^{*}-x^{*}, x-y^{*}\right\rangle \geq 0, \quad \forall x \in K,
\end{aligned}
$$

which has been introduced and studied by Verma [31, 32].

Case 8. If $x^{*}=y^{*}$, then the system (3.12) collapses to the following problem.

Find $x^{*} \in K$ such that

$$
\left\langle T x^{*}, x-x^{*}\right\rangle \geq 0, \quad \forall x \in K \text {. }
$$

Inequality of type (3.13) is called variational inequality, which was introduced and studied by Stampacchia [1] in 1964.

\section{Perturbed Projection Iterative Algorithms}

In this section, by using the projection operator technique, we first verify the equivalence between the extended general nonlinear regularized nonconvex set-valued variational inequalities (3.1) and the fixed point problems. Then, by using the obtained fixed point formulation, we construct two new perturbed projection iterative algorithms with mixed errors for solving the systems (3.1) and (3.6). 
Lemma 4.1. Let $T_{i}, g_{i}, h_{i}(i=1,2), \rho$, and $\eta$ be the same as in the system (3.1). Then $\left(x^{*}, y^{*}, u^{*}, w^{*}\right)$ with $x^{*}, y^{*} \in \mathscr{d l}, h_{1}\left(x^{*}\right), h_{2}\left(y^{*}\right) \in K_{r}$, and $u^{*} \in T_{1}\left(y^{*}, x^{*}\right), w^{*} \in T_{2}\left(x^{*}, y^{*}\right)$ is a solution of the system (3.1), if and only if

$$
\begin{aligned}
& h_{1}\left(x^{*}\right)=P_{K_{r}}\left(g_{1}\left(y^{*}\right)-\rho u^{*}\right), \\
& h_{2}\left(y^{*}\right)=P_{K_{r}}\left(g_{2}\left(x^{*}\right)-\eta w^{*}\right),
\end{aligned}
$$

where $P_{K_{r}}$ is the projection of $\mathscr{d}$ onto the uniformly prox-regular set $K_{r}$.

Proof. Let $\left(x^{*}, y^{*}, u^{*}, w^{*}\right)$ with $x^{*}, y^{*} \in \mathscr{l}, h_{1}\left(x^{*}\right), h_{2}\left(y^{*}\right) \in K_{r}$, and $u^{*} \in T_{1}\left(y^{*}, x^{*}\right), w^{*} \in$ $T_{2}\left(x^{*}, y^{*}\right)$ be a solution of the system (3.1). Then, in view of Lemma 3.1, we have

$$
\begin{gathered}
0 \in \rho u^{*}+h_{1}\left(x^{*}\right)-g_{1}\left(y^{*}\right)+N_{K_{r}}^{P}\left(h_{1}\left(x^{*}\right)\right), \\
0 \in \eta w^{*}+h_{2}\left(y^{*}\right)-g_{2}\left(x^{*}\right)+N_{K_{r}}^{P}\left(h_{2}\left(y^{*}\right)\right),
\end{gathered}
$$

$\Longleftrightarrow$

$$
\begin{aligned}
& g_{1}\left(y^{*}\right)-\rho u^{*} \in\left(I+N_{K_{r}}^{P}\right)\left(h_{1}\left(x^{*}\right)\right), \\
& g_{2}\left(x^{*}\right)-\eta w^{*} \in\left(I+N_{K_{r}}^{P}\right)\left(h_{2}\left(y^{*}\right)\right),
\end{aligned}
$$

$\Longleftrightarrow$

$$
\begin{aligned}
& h_{1}\left(x^{*}\right)=P_{K_{r}}\left(g_{1}\left(y^{*}\right)-\rho u^{*}\right), \\
& h_{2}\left(y^{*}\right)=P_{K_{r}}\left(g_{2}\left(x^{*}\right)-\eta w^{*}\right),
\end{aligned}
$$

where $I$ is identity operator, and we have used the well-known fact that $P_{K_{r}}=\left(I+N_{K_{r}}^{P}\right)^{-1}$.

Remark 4.2. The equality (4.1) can be written as follows:

$$
\begin{array}{cc}
z=g_{1}\left(y^{*}\right)-\rho u^{*}, & t=g_{2}\left(x^{*}\right)-\eta w^{*}, \\
h_{1}\left(x^{*}\right)=P_{K_{r}}(z), & h_{2}\left(y^{*}\right)=P_{K_{r}}(t),
\end{array}
$$

where $\rho, \eta>0$ are two constants.

The fixed point formulation (4.5) enables us to construct the following perturbed iterative algorithms with mixed errors. 
Algorithm 4.3. Let $T_{i}, g_{i}, h_{i}(i=1,2), \rho$, and $\eta$ be the same as in the system (3.1) such that $h_{i}: \mathscr{H} \rightarrow \mathscr{H}$ be an onto operator for $i=1,2$. For arbitrary chosen initial point $\left(z_{0}, t_{0}\right) \in \mathscr{H} \times \mathscr{d}$, compute the iterative sequence $\left\{\left(x_{n}, y_{n}, u_{n}, w_{n}\right)\right\}_{n=0}^{\infty}$ by using

$$
\begin{gathered}
h_{1}\left(x_{n}\right)=P_{K_{r}}\left(z_{n}\right), \quad h_{2}\left(y_{n}\right)=P_{K_{r}}\left(t_{n}\right), \\
z_{n+1}=(1-\alpha) z_{n}+\alpha\left(g_{1}\left(y_{n}\right)-\rho u_{n}+e_{n}\right)+r_{n}, \\
t_{n+1}=(1-\alpha) t_{n}+\alpha\left(g_{2}\left(x_{n}\right)-\eta w_{n}+p_{n}\right)+k_{n}, \\
u_{n} \in T_{1}\left(y_{n}, x_{n}\right), \quad\left\|u_{n}-u_{n+1}\right\| \leq\left(1+(1+n)^{-1}\right) \widehat{H}\left(T_{1}\left(y_{n}, x_{n}\right), T_{1}\left(y_{n+1}, x_{n+1}\right)\right), \\
w_{n} \in T_{2}\left(x_{n}, y_{n}\right), \quad\left\|w_{n}-w_{n+1}\right\| \leq\left(1+(1+n)^{-1}\right) \widehat{H}\left(T_{2}\left(x_{n}, y_{n}\right), T_{2}\left(x_{n+1}, y_{n+1}\right)\right),
\end{gathered}
$$

where initial points $u_{0} \in T_{1}\left(y_{0}, x_{0}\right)$ and $w_{0} \in T_{2}\left(x_{0}, y_{0}\right)$ are chosen arbitrary, $0<\alpha \leq 1$ is a parameter and $\left\{e_{n}\right\}_{n=0}^{\infty},\left\{p_{n}\right\}_{n=0}^{\infty},\left\{r_{n}\right\}_{n=0}^{\infty}$ and $\left\{k_{n}\right\}_{n=0}^{\infty}$ are four sequences in $\mathscr{H}$ to take into account a possible inexact computation of the resolvent operator point satisfying the following conditions:

$$
\begin{gathered}
\lim _{n \rightarrow \infty} e_{n}=\lim _{n \rightarrow \infty} p_{n}=\lim _{n \rightarrow \infty} r_{n}=\lim _{n \rightarrow \infty} k_{n}=0, \\
\sum_{n=1}^{\infty}\left\|e_{n}-e_{n-1}\right\|<\infty, \quad \sum_{n=1}^{\infty}\left\|p_{n}-p_{n-1}\right\|<\infty, \\
\sum_{n=1}^{\infty}\left\|r_{n}-r_{n-1}\right\|<\infty, \quad \sum_{n=1}^{\infty}\left\|k_{n}-k_{n-1}\right\|<\infty .
\end{gathered}
$$

Algorithm 4.4. Let $T_{i}, g_{i}, h_{i}(i=1,2), \rho$, and $\eta$ be the same as in the system (3.6) such that $h_{i}: \mathscr{d} \rightarrow \mathscr{d}$ is an onto operator for $i=1,2$. For arbitrary chosen initial point $\left(z_{0}, t_{0}\right) \in \mathscr{d} \times \mathscr{d}$, compute the iterative sequence $\left\{\left(x_{n}, y_{n}, u_{n}, w_{n}\right)\right\}_{n=0}^{\infty}$ by using

$$
\begin{gathered}
h_{1}\left(x_{n}\right)=P_{K}\left(z_{n}\right), \quad h_{2}\left(y_{n}\right)=P_{K}\left(t_{n}\right), \\
z_{n+1}=(1-\alpha) z_{n}+\alpha\left(g_{1}\left(y_{n}\right)-\rho u_{n}+e_{n}\right)+r_{n}, \\
t_{n+1}=(1-\alpha) t_{n}+\alpha\left(g_{2}\left(x_{n}\right)-\eta w_{n}+p_{n}\right)+k_{n}, \\
u_{n} \in T_{1}\left(y_{n}, x_{n}\right), \quad\left\|u_{n}-u_{n+1}\right\| \leq\left(1+(1+n)^{-1}\right) \widehat{H}\left(T_{1}\left(y_{n}, x_{n}\right), T_{1}\left(y_{n+1}, x_{n+1}\right)\right), \\
w_{n} \in T_{2}\left(x_{n}, y_{n}\right), \quad\left\|w_{n}-w_{n+1}\right\| \leq\left(1+(1+n)^{-1}\right) \widehat{H}\left(T_{2}\left(x_{n}, y_{n}\right), T_{2}\left(x_{n+1}, y_{n+1}\right)\right),
\end{gathered}
$$

where initial points $u_{0} \in T_{1}\left(y_{0}, x_{0}\right)$ and $w_{0} \in T_{2}\left(x_{0}, y_{0}\right)$ are chosen arbitrary, the parameter $\alpha$ and the sequences $\left\{e_{n}\right\}_{n=0}^{\infty},\left\{p_{n}\right\}_{n=0}^{\infty},\left\{r_{n}\right\}_{n=0}^{\infty}$, and $\left\{k_{n}\right\}_{n=0}^{\infty}$ are the same as in Algorithm 4.3. 
Remark 4.5. It should be pointed that

(a) when $r_{n}=k_{n}=0$, for all $n \geq 0$, Algorithms 4.3 and 4.4 reduce to the perturbed iterative process with mean errors;

(b) if $e_{n}=p_{n}=r_{n}=k_{n}=0$, for all $n \geq 0$, then Algorithms 4.3 and 4.4 change into the perturbed iterative process without error.

\section{Main Results}

In this section, we establish the strongly convergence of the sequence generated by the perturbed projection iterative Algorithms 4.3 and 4.4.

Theorem 5.1. Let $T_{i}, g_{i}, h_{i}(i=1,2), \rho$, and $\eta$ be the same as in the system (3.1) such that, for each $i=1,2$,

(a) $T_{i}$ is $\theta_{i}$-strongly monotone with respect to $g_{i}$ and $\gamma_{i}-\widehat{H}$-Lipschitz continuous in the first variable;

(b) $h_{i}$ is $\pi_{i}$-strongly monotone and $\delta_{i}$-Lipschitz continuous;

(c) $g_{i}$ is $\sigma_{i}$-Lipschitz continuous.

If the constants $\rho$ and $\eta$ satisfy the following conditions:

$$
\begin{aligned}
& \left|\rho-\frac{\theta_{1}}{r_{1}^{2}}\right|<\frac{\sqrt{r^{2} \theta_{1}^{2}-r_{1}^{2}\left(r^{2} \sigma_{1}^{2}-\left(r-r^{\prime}\right)^{2}\left(1-\mu_{2}\right)^{2}\right)}}{r r_{1}^{2}}, \\
& \left|\eta-\frac{\theta_{2}}{r_{2}^{2}}\right|<\frac{\sqrt{r^{2} \theta_{2}^{2}-r_{2}^{2}\left(r^{2} \sigma_{2}^{2}-\left(r-r^{\prime}\right)^{2}\left(1-\mu_{1}\right)^{2}\right)}}{r r_{2}^{2}}, \\
& r \theta_{1}>r_{1} \sqrt{r^{2} \sigma_{1}^{2}-\left(r-r^{\prime}\right)^{2}\left(1-\mu_{2}\right)^{2}}, \\
& r \theta_{2}>r_{2} \sqrt{r^{2} \sigma_{2}^{2}-\left(r-r^{\prime}\right)^{2}\left(1-\mu_{1}\right)^{2}}, \\
& r \sigma_{1}>\left(r-r^{\prime}\right)\left(1-\mu_{2}\right), \quad r \sigma_{2}>\left(r-r^{\prime}\right)\left(1-\mu_{1}\right), \\
& \mu_{i}=\sqrt{1-\left(2 \pi_{i}-\delta_{i}^{2}\right)}<1, \quad 2 \pi_{i}<1+\delta_{i}^{2}, \quad(i=1,2),
\end{aligned}
$$

where $r^{\prime} \in(0, r)$, then there exist $x^{*}, y^{*} \in \mathscr{d}$ with $h_{1}\left(x^{*}\right), h_{2}\left(y^{*}\right) \in K_{r}$ and $u^{*} \in T_{1}\left(y^{*}, x^{*}\right)$, $w^{*} \in T_{2}\left(x^{*}, y^{*}\right)$ such that $\left(x^{*}, y^{*}, u^{*}, w^{*}\right)$ is a solution of the system (3.1), and the sequence $\left\{\left(x_{n}, y_{n}, u_{n}, w_{n}\right)\right\}_{n=0}^{\infty}$ generated by Algorithm 4.3 converges strongly to $\left(x^{*}, y^{*}, u^{*}, w^{*}\right)$.

Proof. It follows from (4.6) that

$$
\begin{aligned}
\left\|z_{n+1}-z_{n}\right\| \leq & (1-\alpha)\left\|z_{n}-z_{n-1}\right\|+\alpha\left\|g_{1}\left(y_{n}\right)-g_{1}\left(y_{n-1}\right)-\rho\left(u_{n}-u_{n-1}\right)\right\| \\
& +\alpha\left\|e_{n}-e_{n-1}\right\|+\left\|r_{n}-r_{n-1}\right\| .
\end{aligned}
$$


Since $T_{1}$ is $\theta_{1}$-strongly monotone with respect to $g_{1}$ and $\gamma_{1}-\widehat{H}$-Lipschitz continuous in the first variable and $g_{1}$ is $\sigma_{1}$-Lipschitz continuous, we conclude that

$$
\begin{aligned}
& \left\|g_{1}\left(y_{n}\right)-g_{1}\left(y_{n-1}\right)-\rho\left(u_{n}-u_{n-1}\right)\right\|^{2} \\
& \quad=\left\|g_{1}\left(y_{n}\right)-g_{1}\left(y_{n-1}\right)\right\|^{2}-2 \rho\left\langle u_{n}-u_{n-1}, g_{1}\left(y_{n}\right)-g_{1}\left(y_{n-1}\right)\right\rangle+\rho^{2}\left\|u_{n}-u_{n-1}\right\|^{2} \\
& \quad \leq\left(\sigma_{1}^{2}-2 \rho \theta_{1}\right)\left\|y_{n}-y_{n-1}\right\|^{2}+\rho^{2}\left(1+n^{-1}\right)^{2}\left(\widehat{H}\left(T_{1}\left(y_{n}, x_{n}\right), T_{1}\left(y_{n-1}, x_{n-1}\right)\right)\right)^{2} \\
& \quad \leq\left(\sigma_{1}^{2}-2 \rho \theta_{1}+\rho^{2}\left(1+n^{-1}\right)^{2} r_{1}^{2}\right)\left\|y_{n}-y_{n-1}\right\|^{2} .
\end{aligned}
$$

Substituting (5.3) in (5.2), we get

$$
\begin{aligned}
\left\|z_{n+1}-z_{n}\right\| \leq & (1-\alpha)\left\|z_{n}-z_{n-1}\right\|+\alpha \sqrt{\sigma_{1}^{2}-2 \rho \theta_{1}+\rho^{2}\left(1+n^{-1}\right)^{2} \gamma_{1}^{2}}\left\|y_{n}-y_{n-1}\right\| \\
& +\alpha\left\|e_{n}-e_{n-1}\right\|+\left\|r_{n}-r_{n-1}\right\| .
\end{aligned}
$$

Like the proof (5.4), by using (4.6), we can prove that

$$
\begin{aligned}
\left\|t_{n+1}-t_{n}\right\| \leq & (1-\alpha)\left\|t_{n}-t_{n-1}\right\|+\alpha \sqrt{\sigma_{2}^{2}-2 \eta \theta_{2}+\eta^{2}\left(1+n^{-1}\right)^{2} \gamma_{2}^{2}}\left\|x_{n}-x_{n-1}\right\| \\
& +\alpha\left\|p_{n}-p_{n-1}\right\|+\left\|k_{n}-k_{n-1}\right\| .
\end{aligned}
$$

On the other hand, by using (4.6) and Proposition 2.10, we find that

$$
\begin{aligned}
\left\|x_{n}-x_{n-1}\right\| & \leq\left\|x_{n}-x_{n-1}-\left(h_{1}\left(x_{n}\right)-h_{1}\left(x_{n-1}\right)\right)\right\|+\left\|h_{1}\left(x_{n}\right)-h_{1}\left(x_{n-1}\right)\right\| \\
& =\left\|x_{n}-x_{n-1}-\left(h_{1}\left(x_{n}\right)-h_{1}\left(x_{n-1}\right)\right)\right\|+\left\|P_{K_{r}}\left(z_{n}\right)-P_{K_{r}}\left(z_{n-1}\right)\right\| \\
& \leq\left\|x_{n}-x_{n-1}-\left(h_{1}\left(x_{n}\right)-h_{1}\left(x_{n-1}\right)\right)\right\|+\frac{r}{r-r^{\prime}}\left\|z_{n}-z_{n-1}\right\| .
\end{aligned}
$$

From $\pi_{1}$-strongly monotonicity and $\delta_{1}$-Lipschitz continuity of $h_{1}$, we have

$$
\begin{aligned}
\| x_{n}- & x_{n-1}-\left(h_{1}\left(x_{n}\right)-h_{1}\left(x_{n-1}\right)\right) \|^{2} \\
& =\left\|x_{n}-x_{n-1}\right\|^{2}-2\left\langle h_{1}\left(x_{n}\right)-h_{1}\left(x_{n-1}\right), x_{n}-x_{n-1}\right\rangle+\left\|h_{1}\left(x_{n}\right)-h_{1}\left(x_{n-1}\right)\right\|^{2} \\
& \leq\left(1-2 \pi_{1}+\delta_{1}^{2}\right)\left\|x_{n}-x_{n-1}\right\|^{2} .
\end{aligned}
$$

Substituting (5.7) in (5.6), we obtain

$$
\left\|x_{n}-x_{n-1}\right\| \leq \sqrt{1-2 \pi_{1}+\delta_{1}^{2}}\left\|x_{n}-x_{n-1}\right\|+\frac{r}{r-r^{\prime}}\left\|z_{n}-z_{n-1}\right\|,
$$


which leads to

$$
\left\|x_{n}-x_{n-1}\right\| \leq \frac{r}{\left(r-r^{\prime}\right)\left(1-\sqrt{1-2 \pi_{1}+\delta_{1}^{2}}\right)}\left\|z_{n}-z_{n-1}\right\|
$$

In similar way to the proofs (5.6)-(5.9), we can prove that

$$
\left\|y_{n}-y_{n-1}\right\| \leq \frac{r}{\left(r-r^{\prime}\right)\left(1-\sqrt{1-2 \pi_{2}+\delta_{2}^{2}}\right)}\left\|t_{n}-t_{n-1}\right\|
$$

It follows from (5.4) and (5.10) that

$$
\begin{aligned}
\left\|z_{n+1}-z_{n}\right\| \leq & (1-\alpha)\left\|z_{n}-z_{n-1}\right\|+\alpha \frac{r \sqrt{\sigma_{1}^{2}-2 \rho \theta_{1}+\rho^{2}\left(1+n^{-1}\right)^{2} \gamma_{1}^{2}}}{\left(r-r^{\prime}\right)\left(1-\sqrt{1-2 \pi_{2}+\delta_{2}^{2}}\right)}\left\|t_{n}-t_{n-1}\right\| \\
& +\alpha\left\|e_{n}-e_{n-1}\right\|+\left\|r_{n}-r_{n-1}\right\| .
\end{aligned}
$$

From (5.5) and (5.9), it follows that

$$
\begin{aligned}
\left\|t_{n+1}-t_{n}\right\| \leq & (1-\alpha)\left\|t_{n}-t_{n-1}\right\|+\alpha \frac{r \sqrt{\sigma_{2}^{2}-2 \eta \theta_{2}+\eta^{2}\left(1+n^{-1}\right)^{2} \gamma_{2}^{2}}}{\left(r-r^{\prime}\right)\left(1-\sqrt{1-2 \pi_{1}+\delta_{1}^{2}}\right)}\left\|z_{n}-z_{n-1}\right\| \\
& +\alpha\left\|p_{n}-p_{n-1}\right\|+\left\|k_{n}-k_{n-1}\right\| .
\end{aligned}
$$

Now define $\|\cdot\|_{*}$ on $\mathscr{H} \times \mathscr{H}$ by $\|(x, y)\|_{*}=\|x\|+\|y\|$, for all $(x, y) \in \mathscr{H} \times \mathscr{d}$. It is obvious that $\left(\mathscr{l} \times \mathscr{L},\|\cdot\|_{*}\right)$ is a Hilbert space. Applying (5.11) and (5.12), one has

$$
\begin{aligned}
\left\|\left(z_{n+1}, t_{n+1}\right)-\left(z_{n}, t_{n}\right)\right\|_{*} \leq & (1-\alpha)\left\|\left(z_{n}, t_{n}\right)-\left(z_{n-1}, t_{n-1}\right)\right\|_{*}+\alpha \vartheta(n)\left\|\left(z_{n}, t_{n}\right)-\left(z_{n-1}, t_{n-1}\right)\right\|_{*} \\
& +\alpha\left\|\left(e_{n}, p_{n}\right)-\left(e_{n-1}, p_{n-1}\right)\right\|_{*}+\left\|\left(r_{n}, k_{n}\right)-\left(r_{n-1}, k_{n-1}\right)\right\|_{* \prime}
\end{aligned}
$$

where

$$
\vartheta(n)=\max \left\{\frac{r \sqrt{\sigma_{1}^{2}-2 \rho \theta_{1}+\rho^{2}\left(1+n^{-1}\right)^{2} \gamma_{1}^{2}}}{\left(r-r^{\prime}\right)\left(1-\sqrt{1-2 \pi_{2}+\delta_{2}^{2}}\right)}, \frac{r \sqrt{\sigma_{2}^{2}-2 \eta \theta_{2}+\eta^{2}\left(1+n^{-1}\right)^{2} \gamma_{2}^{2}}}{\left(r-r^{\prime}\right)\left(1-\sqrt{1-2 \pi_{1}+\delta_{1}^{2}}\right)}\right\} .
$$


Obviously, $\vartheta(n) \rightarrow \vartheta$, as $n \rightarrow \infty$, where

$$
\vartheta=\max \left\{\frac{r \sqrt{\sigma_{1}^{2}-2 \rho \theta_{1}+\rho^{2} \gamma_{1}^{2}}}{\left(r-r^{\prime}\right)\left(1-\sqrt{1-2 \pi_{2}+\delta_{2}^{2}}\right)}, \frac{r \sqrt{\sigma_{2}^{2}-2 \eta \theta_{2}+\eta^{2} \gamma_{2}^{2}}}{\left(r-r^{\prime}\right)\left(1-\sqrt{1-2 \pi_{1}+\delta_{1}^{2}}\right)}\right\} .
$$

In view of the condition (5.1), we know that $0 \leq \vartheta<1$. Then, for $\widehat{\vartheta}=(1 / 2)(\vartheta+1) \in(\vartheta, 1)$, there exists $n_{0} \geq 1$ such that $\vartheta(n)<\widehat{\vartheta}$ for each $n \geq n_{0}$. Thus, it follows from (5.13) that, for each $n \geq n_{0}$,

$$
\begin{aligned}
\|\left(z_{n+1},\right. & \left.t_{n+1}\right)-\left(z_{n}, t_{n}\right) \|_{*} \\
\leq & (1-\alpha)\left\|\left(z_{n}, t_{n}\right)-\left(z_{n-1}, t_{n-1}\right)\right\|_{*}+\alpha \widehat{\vartheta}\left\|\left(z_{n}, t_{n}\right)-\left(z_{n-1}, t_{n-1}\right)\right\|_{*} \\
& +\alpha\left\|\left(e_{n}, p_{n}\right)-\left(e_{n-1}, p_{n-1}\right)\right\|_{*}+\left\|\left(r_{n}, k_{n}\right)-\left(r_{n-1}, k_{n-1}\right)\right\|_{*} \\
= & (1-\alpha(1-\widehat{\vartheta}))\left\|\left(z_{n}, t_{n}\right)-\left(z_{n-1}, t_{n-1}\right)\right\|_{*}+\alpha\left\|\left(e_{n}, p_{n}\right)-\left(e_{n-1}, p_{n-1}\right)\right\|_{*} \\
& +\left\|\left(r_{n}, k_{n}\right)-\left(r_{n-1}, k_{n-1}\right)\right\|_{*} \\
\leq & (1-\alpha(1-\widehat{\vartheta}))\left[(1-\alpha(1-\widehat{\vartheta}))\left\|\left(z_{n-1}, t_{n-1}\right)-\left(z_{n-2}, t_{n-2}\right)\right\|_{*}\right. \\
& +\alpha\left\|\left(e_{n}, p_{n}\right)-\left(e_{n-1}, p_{n-1}\right)\right\|_{*}+\left\|\left(r_{n}, k_{n}\right)-\left(r_{n-1}, k_{n-1}\right)\right\|_{*} \\
= & (1-\alpha(1-\widehat{\vartheta}))^{2}\left\|\left(z_{n-1}, t_{n-1}\right)-\left(z_{n-2}, t_{n-2}\right)\right\|_{*} \\
& +\alpha\left[(1-\alpha(1-\widehat{\vartheta}))\left\|\left(e_{n-1}, p_{n-1}\right)-\left(e_{n-2}, p_{n-2}\right)\right\|_{*}+\left\|\left(e_{n}, p_{n}\right)-\left(e_{n-1}, p_{n-1}\right)\right\|_{*}\right] \\
& +(1-\alpha(1-\widehat{\vartheta}))\left\|\left(r_{n-1}, k_{n-1}\right)-\left(r_{n-2}, k_{n-2}\right)\right\|_{*}+\left\|\left(r_{n}, k_{n}\right)-\left(r_{n-1}, k_{n-1}\right)\right\|_{*} \\
\leq & \\
& \vdots \\
\leq & (1-\alpha(1-\widehat{\vartheta}))^{n-n_{0}}\left\|\left(z_{n_{0}+1}, t_{n_{0}+1}\right)-\left(z_{n 0}, t_{n_{0}}\right)\right\|_{*} \\
& +\alpha \sum_{i=1}^{n-n_{0}}(1-\alpha(1-\widehat{\vartheta}))^{i-1}\left\|\left(r_{n-(i-1)}, k_{n-(i-1)}\right)-\left(r_{n-i}, k_{n-i}\right)\right\|_{*} \cdot \\
& \\
& \\
& \\
& \\
&
\end{aligned}
$$


Hence, for any $m \geq n>n_{0}$, we have

$$
\begin{aligned}
\left\|\left(z_{m}, t_{m}\right)-\left(z_{n}, t_{n}\right)\right\|_{*} \leq & \sum_{j=n}^{m-1}\left\|\left(z_{j+1}, t_{j+1}\right)-\left(z_{j}, t_{j}\right)\right\|_{*} \\
\leq & \sum_{j=n}^{m-1}(1-\alpha(1-\widehat{\vartheta}))^{j-n_{0}}\left\|\left(z_{n_{0}+1}, t_{n_{0}+1}\right)-\left(z_{n_{0}}, t_{n_{0}}\right)\right\|_{*} \\
& +\alpha \sum_{j=n}^{m-1} \sum_{i=1}^{j-n_{0}}(1-\alpha(1-\widehat{\vartheta}))^{i-1}\left\|\left(e_{n-(i-1)}, p_{n-(i-1)}\right)-\left(e_{n-i}, p_{n-i}\right)\right\|_{*} \\
& +\sum_{j=n}^{m-1} \sum_{i=1}^{j-n_{0}}(1-\alpha(1-\widehat{\vartheta}))^{i-1}\left\|\left(r_{n-(i-1)}, k_{n-(i-1)}\right)-\left(r_{n-i}, k_{n-i}\right)\right\|_{*} .
\end{aligned}
$$

Since $1-\alpha(1-\widehat{\vartheta}) \in(0,1)$, it follows from (4.7) and (5.17) that $\left\|\left(z_{m}, t_{m}\right)-\left(z_{n}, t_{n}\right)\right\|_{*}=\| z_{m}-$ $z_{n}\|+\| t_{m}-t_{n} \| \rightarrow 0$, as $n \rightarrow \infty$. Hence, $\left\{z_{n}\right\}$ and $\left\{t_{n}\right\}$ are both Cauchy sequences in $\mathscr{L}$, and so there exist $z^{*}$ and $t^{*} \in \mathscr{l}$ such that $z_{n} \rightarrow z^{*}$ and $t_{n} \rightarrow t^{*}$, as $n \rightarrow \infty$. By the inequalities (5.9) and (5.10), it follows that the sequences $\left\{x_{n}\right\}$ and $\left\{y_{n}\right\}$ are both also Cauchy in $\mathscr{L}$. Thus, there exist $x^{*}, y^{*} \in \mathscr{H}$ such that $x_{n} \rightarrow x^{*}$ and $y_{n} \rightarrow y^{*}$, as $n \rightarrow \infty$. Since for $i=1,2, T_{i}$ is $\gamma_{i}-\widehat{H}$-Lipschitz continuous in the first variable, it follows from (4.6) that

$$
\begin{aligned}
\left\|u_{n}-u_{n+1}\right\| & \leq\left(1+(1+n)^{-1}\right) \widehat{H}\left(T_{1}\left(y_{n}, x_{n}\right), T_{1}\left(y_{n+1}, x_{n+1}\right)\right) \\
& \leq\left(1+(1+n)^{-1}\right) \gamma_{1}\left\|y_{n}-y_{n+1}\right\| \longrightarrow 0, \\
\left\|w_{n}-w_{n+1}\right\| & \leq\left(1+(1+n)^{-1}\right) \widehat{H}\left(T_{2}\left(x_{n}, y_{n}\right), T_{2}\left(x_{n+1}, y_{n+1}\right)\right) \\
& \leq\left(1+(1+n)^{-1}\right) \gamma_{2}\left\|x_{n}-x_{n+1}\right\| \longrightarrow 0,
\end{aligned}
$$

as $n \rightarrow \infty$. Hence, $\left\{u_{n}\right\}$ and $\left\{w_{n}\right\}$ are also both Cauchy sequences in $\mathscr{t}$ and so there exist $u^{*}, w^{*} \in \mathscr{L}$ such that $u_{n} \rightarrow u^{*}$ and $w_{n} \rightarrow w^{*}$, as $n \rightarrow \infty$. Further, noting $u_{n} \in T_{1}\left(y_{n}, x_{n}\right)$, we have

$$
\begin{aligned}
d\left(u^{*}, T_{1}\left(y^{*}, x^{*}\right)\right) & =\inf \left\{\left\|u^{*}-q\right\|: q \in T_{1}\left(y^{*}, x^{*}\right)\right\} \\
& \leq\left\|u^{*}-u_{n}\right\|+d\left(u_{n}, T_{1}\left(y^{*}, x^{*}\right)\right) \\
& \leq\left\|u^{*}-u_{n}\right\|+\widehat{H}\left(T_{1}\left(y_{n}, x_{n}\right), T_{1}\left(y^{*}, x^{*}\right)\right) \\
& \leq\left\|u^{*}-u_{n}\right\|+\gamma_{1}\left\|y_{n}-y^{*}\right\| .
\end{aligned}
$$

Since $w_{n} \in T_{2}\left(x_{n}, y_{n}\right)$, like the proof (5.19), we obtain

$$
d\left(w^{*}, T_{2}\left(x^{*}, y^{*}\right)\right) \leq\left\|w^{*}-w_{n}\right\|+\gamma_{2}\left\|x_{n}-x^{*}\right\| .
$$


The right sides of the inequalities (5.19) and (5.20) tend to zero as $n \rightarrow \infty$. Hence, $u^{*} \in$ $T_{1}\left(y^{*}, x^{*}\right)$ and $w^{*} \in T_{2}\left(x^{*}, y^{*}\right)$. Since the operators $g_{1}$ and $g_{2}$ are continuous, it follows from (4.6) and (4.7) that

$$
z^{*}=g_{1}\left(y^{*}\right)-\rho u^{*}, \quad t^{*}=g_{2}\left(x^{*}\right)-\eta w^{*}
$$

Since the operators $h_{1}, h_{2}$, and $P_{K_{r}}$ are continuous, it follows from (4.6) and (5.21) that

$$
h_{1}\left(x^{*}\right)=P_{K_{r}}\left(z^{*}\right)=P_{K_{r}}\left(g_{1}\left(y^{*}\right)-\rho u^{*}\right), \quad h_{2}\left(y^{*}\right)=P_{K_{r}}\left(t^{*}\right)=P_{K_{r}}\left(g_{2}\left(x^{*}\right)-\eta w^{*}\right) .
$$

Now, Lemma 4.1 guarantees that $\left(x^{*}, y^{*}, u^{*}, w^{*}\right)$ is a solution of the system (3.1). This completes the proof.

Theorem 5.2. Let $T_{i}, g_{i}, h_{i}(i=1,2), \rho$, and $\eta$ be the same as in the system (3.6) such that, for each $i=1,2$,

(a) $T_{i}$ is $\theta_{i}$-strongly monotone with respect to $g_{i}$ and $\gamma_{i}-\widehat{H}$-Lipschitz continuous in the first variable;

(b) $h_{i}$ is $\pi_{i}$-strongly monotone and $\delta_{i}$-Lipschitz continuous;

(c) $g_{i}$ is $\sigma_{i}$-Lipschitz continuous.

If the constants $\rho$ and $\eta$ satisfy the following conditions:

$$
\begin{gathered}
\left|\rho-\frac{\theta_{1}}{r_{1}^{2}}\right|<\frac{\sqrt{\theta_{1}^{2}-r_{1}^{2}\left(\sigma_{1}^{2}-\left(1-\mu_{2}\right)^{2}\right)}}{r_{1}^{2}}, \\
\left|\eta-\frac{\theta_{2}}{r_{2}^{2}}\right|<\frac{\sqrt{\theta_{2}^{2}-r_{2}^{2}\left(\sigma_{2}^{2}-\left(1-\mu_{1}\right)^{2}\right)}}{r_{2}^{2}}, \\
\theta_{1}>\gamma_{1} \sqrt{\sigma_{1}^{2}-\left(1-\mu_{2}\right)^{2}}, \\
\theta_{2}>\gamma_{2} \sqrt{\sigma_{2}^{2}-\left(1-\mu_{1}\right)^{2}}, \\
\sigma_{1}+\mu_{2}>1, \quad \sigma_{2}+\mu_{1}>1, \quad(i=1,2),
\end{gathered}
$$

then there exist $x^{*}, y^{*} \in \mathscr{H}$ with $h_{1}\left(x^{*}\right), h_{2}\left(y^{*}\right) \in K$ and $u^{*} \in T_{1}\left(y^{*}, x^{*}\right), w^{*} \in T_{2}\left(x^{*}, y^{*}\right)$ such that $\left(x^{*}, y^{*}, u^{*}, w^{*}\right)$ is a solution of the system (3.6) and the sequence $\left\{\left(x_{n}, y_{n}, u_{n}, w_{n}\right)\right\}_{n=0}^{\infty}$ generated by Algorithm 4.4 converges strongly to $\left(x^{*}, y^{*}, u^{*}, w^{*}\right)$.

Remark 5.3. Using the method presented in this paper, one can extend Theorems 5.1 and 5.2 to a system of $n$-generalized variational inequalities. 


\section{Acknowledgments}

The authors are very grateful to the referees for their comments and suggestions which improved the presentation of this paper. The work was supported by the Basic Science Research Program through the National Research Foundation of Korea (NRF) funded by the Ministry of Education, Science and Technology (Grant number: 2011-0021821).

\section{References}

[1] G. Stampacchia, "Formes bilinéaires coercitives sur les ensembles convexes," vol. 258, pp. 4413-4416, 1964.

[2] A. Bensoussan and J.-L. Lions, Applications des Inéquations Variationnelles en Contrôle Stochastique, Dunod, Paris, France, 1978.

[3] R. Glowinski and P. Letallec, Augmented Lagrangian and Operator-Splitting Methods in Nonlinear Mechanics, vol. 9 of SIAM Studies in Applied Mathematics, Society for Industrial and Applied Mathematics (SIAM), Philadelphia, Pa, USA, 1989.

[4] P. T. Harker and J.-S. Pang, "Finite-dimensional variational inequality and nonlinear complementarity problems: a survey of theory, algorithms and applications," Mathematical Programming, vol. 48, no. 2, pp. 161-220, 1990.

[5] R. P. Agarwal, Y. J. Cho, and N. Petrot, "Systems of general nonlinear set-valued mixed variational inequalities problems in Hilbert spaces," Fixed Point Theory and Applications, vol. 2011, article 31, 2011.

[6] M. Alimohammady, J. Balooee, Y. J. Cho, and M. Roohi, "New perturbed finite step iterative algorithms for a system of extended generalized nonlinear mixed quasi-variational inclusions," Computers $\mathcal{E}$ Mathematics with Applications, vol. 60, no. 11, pp. 2953-2970, 2010.

[7] M. Alimohammady, J. Balooee, Y. J. Cho, and M. Roohi, "Iterative algorithms for a new class of extended general nonconvex set-valued variational inequalities," Nonlinear Analysis: Theory, Methods E Applications A, vol. 73, no. 12, pp. 3907-3923, 2010.

[8] A. Bnouhachem and M. A. Noor, "Numerical method for general mixed quasi-variational inequalities," Applied Mathematics and Computation, vol. 204, no. 1, pp. 27-36, 2008.

[9] M. Bounkhel, "Existence results of nonconvex differential inclusions," Portugaliae Mathematica. Nova Série, vol. 59, no. 3, pp. 283-309, 2002.

[10] M. Bounkhel, "General existence results for second order nonconvex sweeping process with unbounded perturbations," Portugaliae Mathematica: Nova Série, vol. 60, no. 3, pp. 269-304, 2003.

[11] M. Bounkhel and D.-L. Azzam, "Existence results on the second-order nonconvex sweeping processes with perturbations," Set-Valued Analysis, vol. 12, no. 3, pp. 291-318, 2004.

[12] M. Bounkhel, L. Tadj, and A. Hamdi, "Iterative schemes to solve nonconvex variational problems," Journal of Inequalities in Pure and Applied Mathematics, vol. 4, pp. 1-14, 2003.

[13] M. Bounkhel and L. Thibault, "Further characterizations of regular sets in Hilbert spaces and their applications to nonconvex sweeping process," Preprint, Centro de Modelamiento Matematico (CMM), Universidad de Chile, 2000.

[14] A. Canino, "On p-convex sets and geodesics," Journal of Differential Equations, vol. 75, no. 1, pp. 118$157,1988$.

[15] S. S. Chang, H. W. Joseph Lee, and C. K. Chan, "Generalized system for relaxed cocoercive variational inequalities in Hilbert spaces," Applied Mathematics Letters, vol. 20, no. 3, pp. 329-334, 2007.

[16] Y. J. Cho and S. Wang, "An iterative scheme with a countable family of nonexpansive mappings for variational inequality problems in Hilbert spaces," Journal of Inequalities and Applications, vol. 2010, Article ID 687374, 14 pages, 2010.

[17] Y. J. Cho, I. K. Argyros, and N. Petrot, "Approximation methods for common solutions of generalized equilibrium, systems of nonlinear variational inequalities and fixed point problems," Computers $\mathcal{E}$ Mathematics with Applications, vol. 60, no. 8, pp. 2292-2301, 2010.

[18] Y. J. Cho and N. Petrot, "Regularization and iterative method for general variational inequality problem in Hilbert spaces," Journal of Inequalities and Applications, vol. 2011, article 21, 2011.

[19] F. H. Clarke, Optimization and Nonsmooth Analysis, Canadian Mathematical Society Series of Monographs and Advanced Texts, Wiley-Interscience, New York, NY, USA, 1983.

[20] F. H. Clarke, Yu. S. Ledyaev, R. J. Stern, and P. R. Wolenski, Nonsmooth Analysis and Control Theory, vol. 178 of Graduate Texts in Mathematics, Springer, New York, NY, USA, 1998. 
[21] F. H. Clarke, R. J. Stern, and P. R. Wolenski, "Proximal smoothness and the lower $C^{2}$ property," Journal of Convex Analysis, vol. 2, no. 1-2, pp. 117-144, 1995.

[22] Z. Huang and M. A. Noor, "An explicit projection method for a system of nonlinear variational inequalities with different $(\gamma, r)$-cocoercive mappings," Applied Mathematics and Computation, vol. 190, no. 1, pp. 356-361, 2007.

[23] J.-L. Lions and G. Stampacchia, "Variational inequalities," Communications on Pure and Applied Mathematics, vol. 20, pp. 493-519, 1967.

[24] A. Moudafi, "An algorithmic approach to prox-regular variational inequalities," Applied Mathematics and Computation, vol. 155, no. 3, pp. 845-852, 2004.

[25] M. A. Noor, "Iterative schemes for nonconvex variational inequalities," Journal of Optimization Theory and Applications, vol. 121, no. 2, pp. 385-395, 2004.

[26] M. A. Noor, Variational Inequalities and Applications, Lecture Notes, Mathematics Department, COMSATS Institute of information Technology, Islamabad, Pakistan, 2007-2009.

[27] L.-P. Pang, J. Shen, and H.-S. Song, "A modified predictor-corrector algorithm for solving nonconvex generalized variational inequality," Computers E Mathematics with Applications, vol. 54, no. 3, pp. 319325, 2007.

[28] R. A. Poliquin, R. T. Rockafellar, and L. Thibault, "Local differentiability of distance functions," Transactions of the American Mathematical Society, vol. 352, no. 11, pp. 5231-5249, 2000.

[29] X. Qin, Y. J. Cho, and S. M. Kang, "Convergence analysis on hybrid projection algorithms for equilibrium problems and variational inequality problems," Mathematical Modelling and Analysis, vol. 14, no. 3, pp. 335-351, 2009.

[30] X. Qin, J. I. Kang, and Y. J. Cho, “On quasi-variational inclusions and asymptotically strict pseudocontractions," Journal of Nonlinear and Convex Analysis, vol. 11, no. 3, pp. 441-453, 2010.

[31] R. U. Verma, "Projection methods, algorithms, and a new system of nonlinear variational inequalities," Computers \& Mathematics with Applications, vol. 41, no. 7-8, pp. 1025-1031, 2001.

[32] R. U. Verma, "General convergence analysis for two-step projection methods and applications to variational problems," Applied Mathematics Letters, vol. 18, no. 11, pp. 1286-1292, 2005.

[33] R. U. Verma, "Generalized system for relaxed cocoercive variational inequalities and projection methods," Journal of Optimization Theory and Applications, vol. 121, no. 1, pp. 203-210, 2004.

[34] H. Yang, L. Zhou, and Q. Li, "A parallel projection method for a system of nonlinear variational inequalities," Applied Mathematics and Computation, vol. 217, no. 5, pp. 1971-1975, 2010.

[35] Y. Yao, Y. J. Cho, and Y.-C. Liou, "Iterative algorithms for hierarchical fixed points problems and variational inequalities," Mathematical and Computer Modelling, vol. 52, no. 9-10, pp. 1697-1705, 2010.

[36] Y. Yao, Y. J. Cho, and Y.-C. Liou, "Iterative algorithms for variational inclusions, mixed equilibrium and fixed point problems with application to optimization problems," Central European Journal of Mathematics, vol. 9, no. 3, pp. 640-656, 2011.

[37] Y. Yao, Y. J. Cho, and Y.-C. Liou, "Algorithms of common solutions for variational inclusions, mixed equilibrium problems and fixed point problems," European Journal of Operational Research, vol. 212, no. 2, pp. 242-250, 2011. 


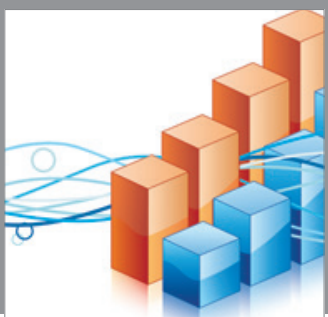

Advances in

Operations Research

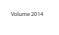

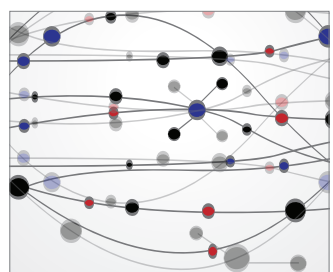

\section{The Scientific} World Journal
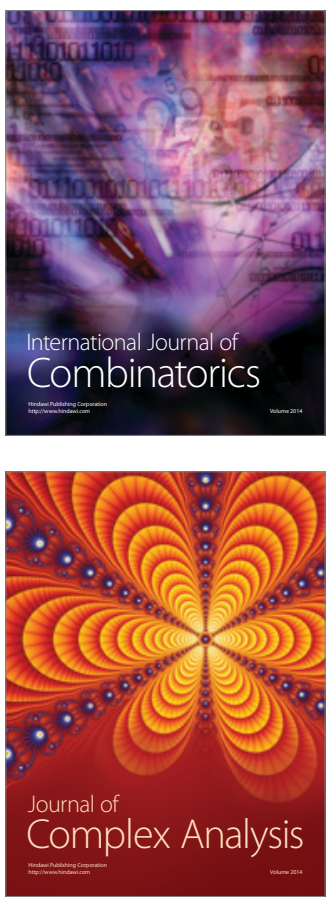

International Journal of

Mathematics and

Mathematical

Sciences
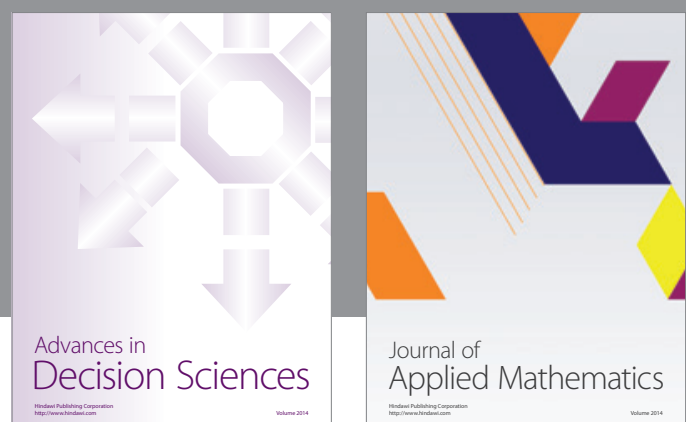

Journal of

Applied Mathematics
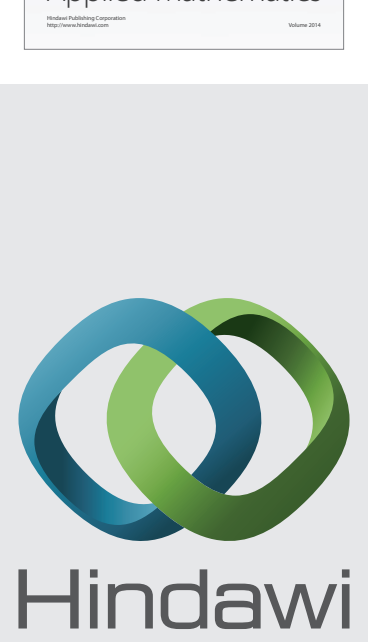

Submit your manuscripts at http://www.hindawi.com
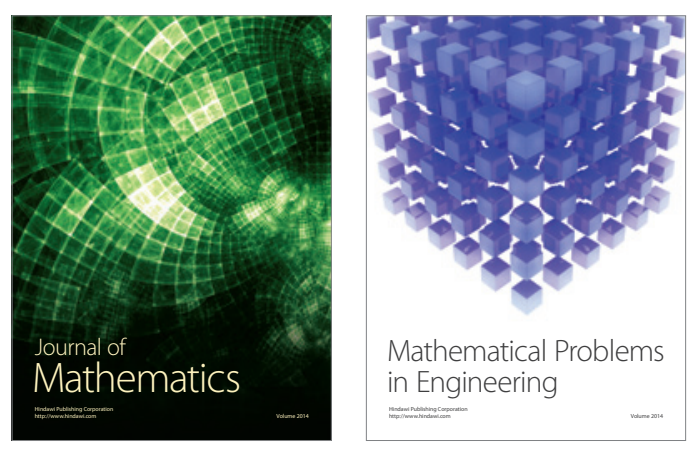

Mathematical Problems in Engineering
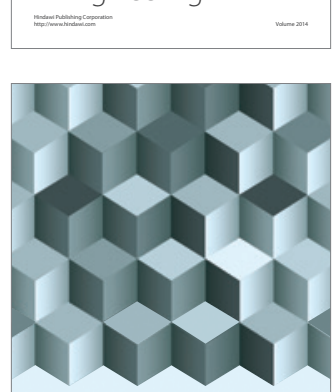

Journal of

Function Spaces
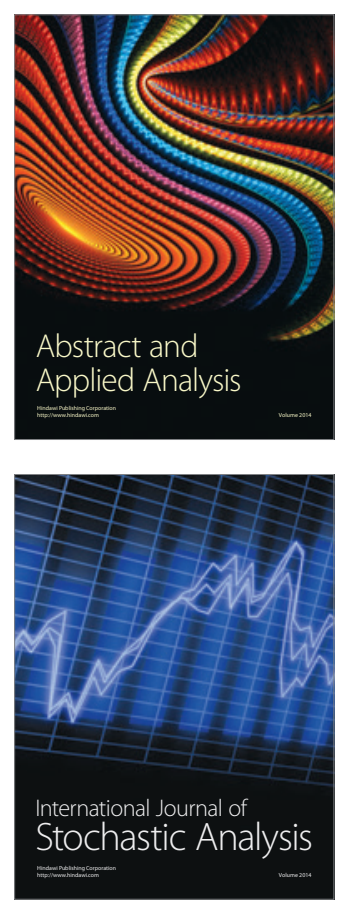

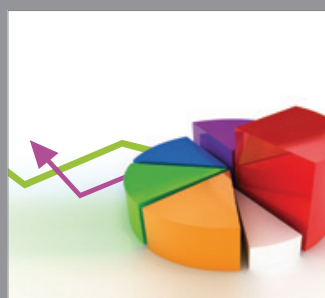

ournal of

Probability and Statistics

Promensencen
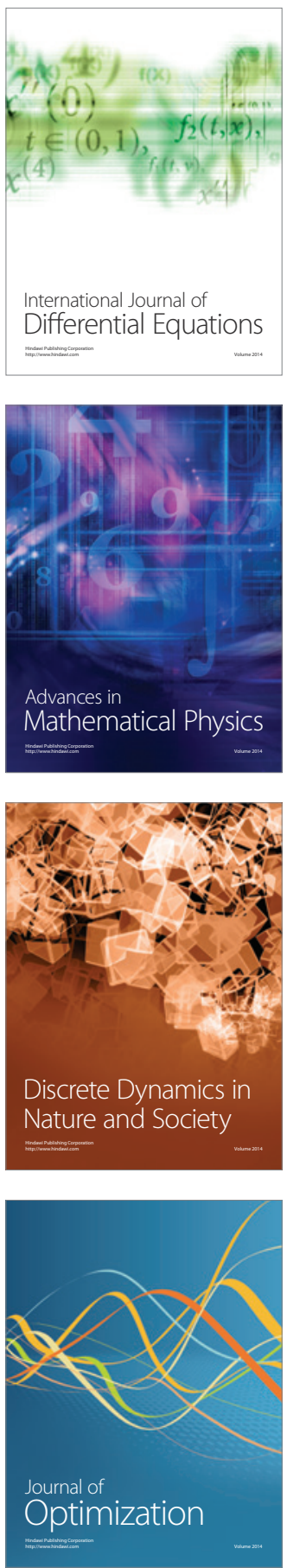